(C) 2011 IEEE. Reprinted, with permission, from Youguang Guo, Analysis of Transient Overvoltage in $220 \mathrm{kV}$ Saturated Core HTS FCL , Magnetics, IEEE Transactions on, October 2011. This material is posted here with permission of the IEEE. Such permission of the IEEE does not in any way imply IEEE endorsement of any of the University of Technology, Sydney's products or services. Internal or personal use of this material is permitted.

However, permission to reprint/republish this material for advertising or promotional purposes or for creating new collective works for resale or redistribution must be obtained from the IEEE by writing to pubs-permissions@ieee.org. By choosing to view this document, you agree to all provisions of the copyright laws protecting it 


\title{
Analysis of Transient Overvoltage in 220kV Saturated Core HTS FCL
}

\author{
Hongli Xiao ${ }^{1}$, Jie Qiu ${ }^{1}$, Shuhong Wang ${ }^{1}$, Qiuhui Zhang ${ }^{1}$, Weizhi Gong ${ }^{2}$, Ying Xin², Jian Guo Zhu ${ }^{3}$ Youguang Guo ${ }^{3}$ \\ ${ }^{1}$ State Key laboratory of Electrical Insulation and Power Equipment, Faculty of Electrical Engineering, Xi'an Jiaotong \\ University, 28 West Xian Ning Road, Xi’an, 710049, China \\ ${ }^{2}$ Innopower Superconductor Cable Co., Ltd, Beijing, 100176, China \\ ${ }^{3}$ Faculty of Engineering and Information Technology, University of Technology, Sydney, P.O. Box 123, Broadway, NSW2007, \\ Australia
}

Saturated core high temperature superconducting (HTS) fault current limiter (FCL) is one kind of limiter that can work effectively on short-circuit current limitation. In this paper, an equivalent circuit model depending on AC winding of HTS FCL is built for voltage distribution analysis under the pulsed voltage. The equivalent circuit components, such as the capacitances and inductances, are calculated by using finite element method. The voltage distribution and oscillation analysis of AC winding may benefit to the insulation design of HTS FCL.

\section{Index Terms - High temperature superconductor fault current limiter (HTS FCL), transient overvoltage, lightning wave}

\section{INTRODUCTION}

$\mathrm{T}^{\mathrm{s}}$ The capacity of power system is increasing so as to the capacity of short circuit. Short-circuit current may exceed the interrupting capacity of breaker. It is an emergency to take some steps for short-circuit current limitation. The Saturated core HTS FCL will be one kind of innovative protection apparatus for high voltage power grids. It works automatically; furthermore, it offers many advantages: rapid reaction to fault current, low impedance in normal conditions, and large impedance during fault conditions [1-3].

Voltage distribution of AC windings of HTS FCL is extremely uneven under the condition of impulse voltage excitation and high-frequency voltage oscillation is occurred, and then may destroy the AC winding insulation. In this paper, voltage distribution and its oscillation of HTS FCL are discussed under the lightning-impulse voltage. The insulation property may be verified by imposing the impulse electrical potential on each pancake of AC winding

\section{LUMPED PARAMETER MODEL UNDER TRANSIENT OVERVOLTAGE}

Fig. 1 shows the schematic of single-phase saturated core HTS FCL, which is composed of two magnetic cores, two AC windings and a superconducting DC bias coil. The permeability of the magnetic core is nonlinear. At the normal operation, both magnetic cores are driven into saturation by the DC bias. As saturated core FCL is saturated, the impedance of the FCL is very low. When a short-circuit fault occurs, rapid increased AC current drives both magnetic cores out of saturation alternatively. Hence, the cores of FCL alternately work in linearity. The impedance of the FCL becomes large to limit the fault current.

Each of two AC windings of the same phase has the same structure with 60 turns and 36 pancakes. In order to analyze transient impulse voltage distribution, the $\mathrm{AC}$ winding needs to be subdivided into several units as shown in Fig. 2 (a). Each unit contains a longitudinal capacitance $C_{s}$, a grounding capacitance $C_{g}$ and an inductance $L$. The equivalent circuit model is shown in Fig. 2 (b) [4-6]. Since the turn number of each pancake is less than 2, calculation based on one pancake will get higher accuracy.

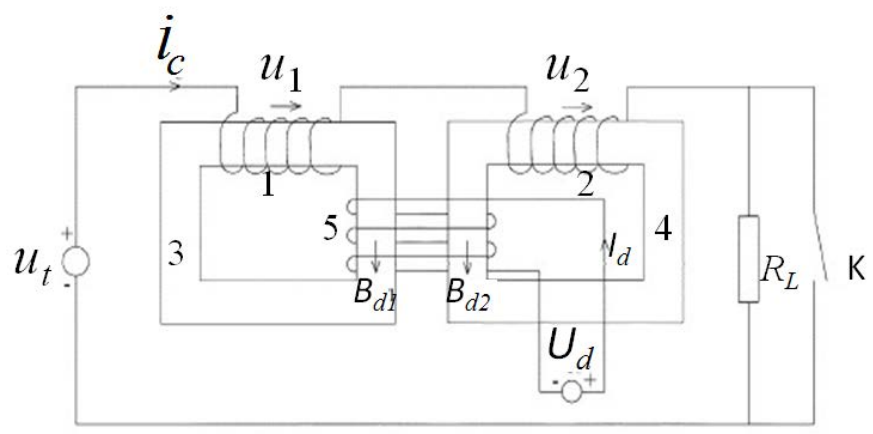

Fig. 1. Experimental circuit for a one phase saturated core FCL. 1, 2: AC windings. 3, 4: Magnetic cores.5: Superconducting DC coils

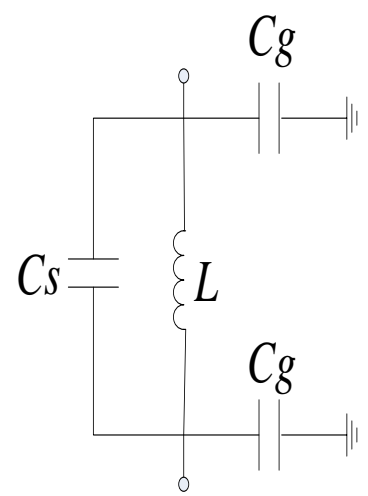

(a) Each unit

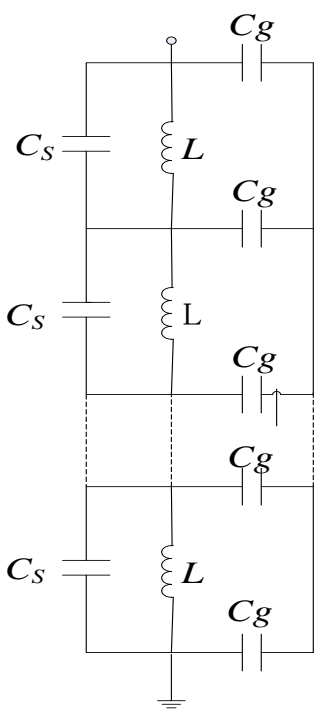

(b) Equivalent circuit
Fig. 2. Equivalent circuit of FCL AC windings under impulse overvoltage excitation 


\section{CAlCulation of EQuivalent PaRAmeters}

The distributed and stray capacitances of the AC coil in HTS FCL have a substantive effect on the transient voltage distribution in the coil. The distribution of the initial voltage in the coil is determined by the distributed capacitance when the steep-front impulse voltage invades the coil. However, the final voltage distribution of the coil is determined by the inductance distribution. The disparity of the initial voltage and the final voltage leads to voltage oscillation. Therefore, the accurate capacitance and inductance calculation model for the coils is crucial to compute the impulse voltage distribution.

\section{A. Calculation of capacitance parameters}

There are two kinds of capacitances in the equivalent circuit. One is longitudinal equivalent capacitance, and another is grounding capacitance. The longitudinal capacitance expresses the electric field effect between the turn-to-turn capacitance and the pancake capacitance, while the grounding capacitance indicates the electric field effect between the winding with ground, such as grounded core.

In this paper, the equivalent capacitance parameters are calculated by finite element analysis (FEA) method. Because the number of turns per pancake is not an integer, the 3 dimensional (3D) FEA model of the coil should be built to analyze the capacitance parameter. Fig. 3 shows the 3D FEA model for calculating the capacitance parameters.

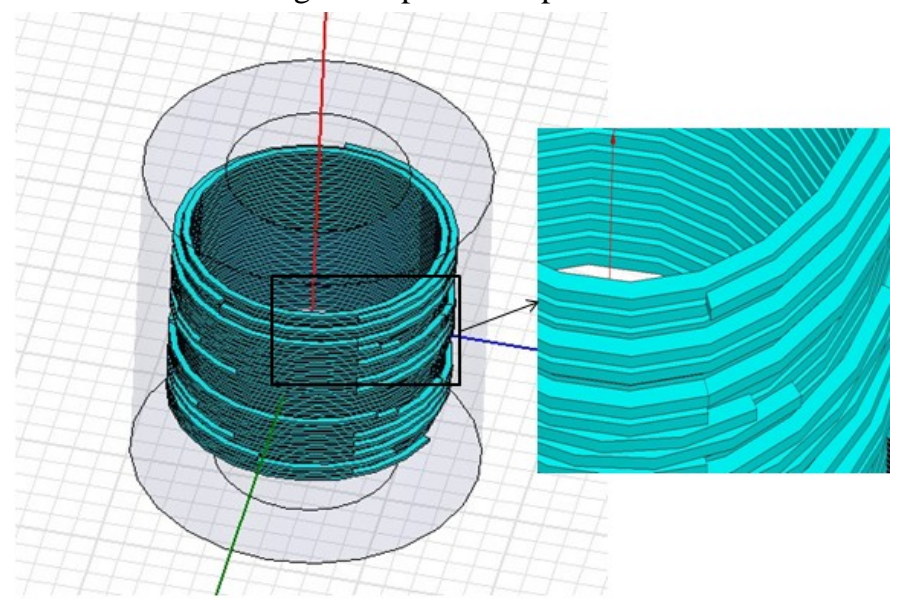

Fig. 3. Model of capacitance Calculation

Fig. 4 shows the capacitances between the first with other pancakes. According to Fig. 4, obviously, the pancake capacitance is dominated by the capacitance between two adjacent connected pancakes, and the capacitances of the unconnected pancakes are too small to take into consideration. The grounding capacitances of the pancakes are depicted in Fig. 5. From Fig.4 and Fig.5, grounding capacitance is about 10 percent of capacitance between adjacent pancakes. Therefore, the capacitance between pancakes has a major effect on the initial voltage distribution in coils under transient impulse overvoltage.

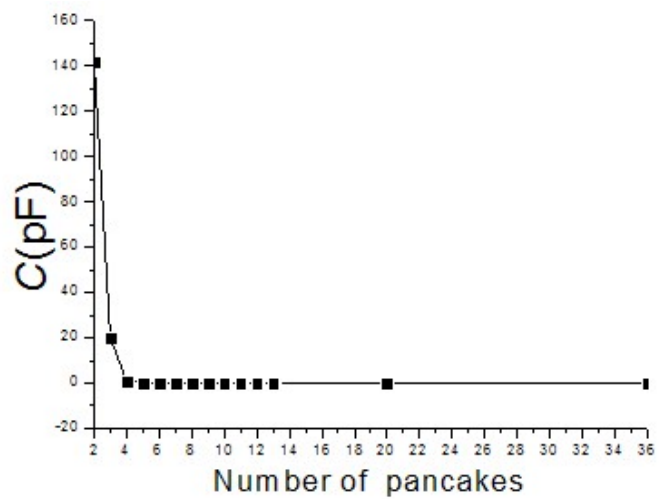

Fig. 4. Capacitances between the first and other pancakes

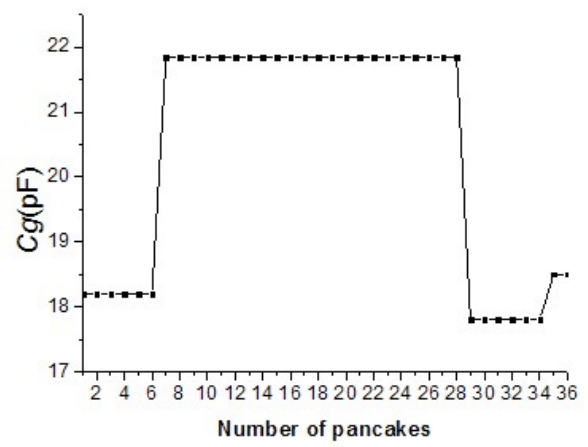

Fig. 5. Grounding capacitances of the pancakes

\section{B. Calculation of inductance}

A single-phase AC winding consists of two coils with the same structure and size. The two coils are wounded on separate cores, respectively. The mutual inductance of these two coils could be ignored. However, the self-inductance of each pancake and mutual inductances among pancakes in the coil should be considered. 2D axisymmetric magnetic field is adopted for analysis. In the case of very fast transient overvoltage, due to skin effect, the core can be ignored [4].

The self- and mutual inductance of the pancakes in FCL AC winding are calculated using the Energy Perturbation method based on FEA [3]. Fig. 6 shows the FEA model of inductance calculation.

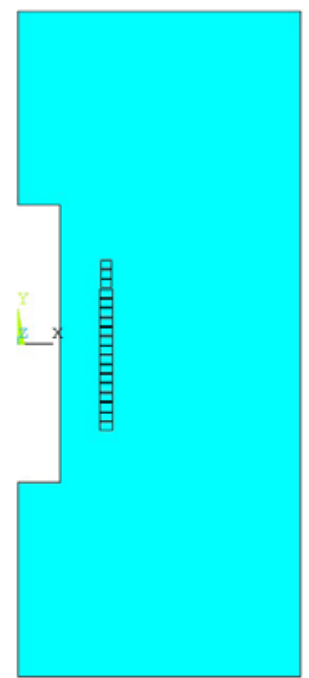

Fig. 6. Model of Inductance Calculation 
Fig. 7 shows the mutual inductances between the 10th and other pancakes, According to Fig.7, The mutual-inductances should be considered Fig. 8 shows the self-inductances of all units.

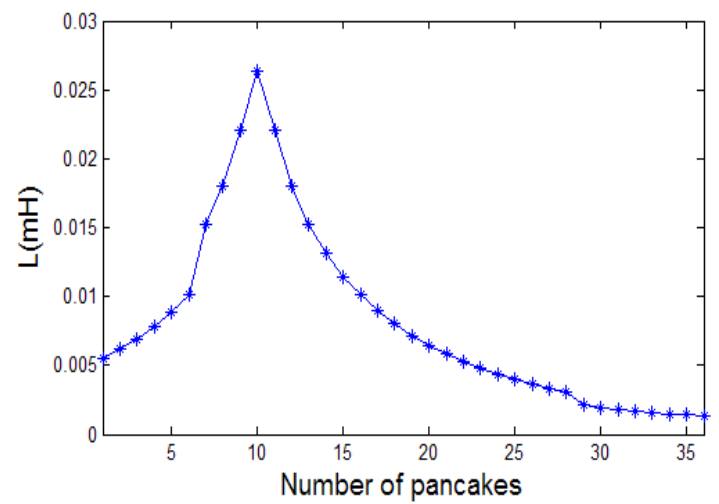

Fig. 7. Mutual inductances between the 10th and other pancakes

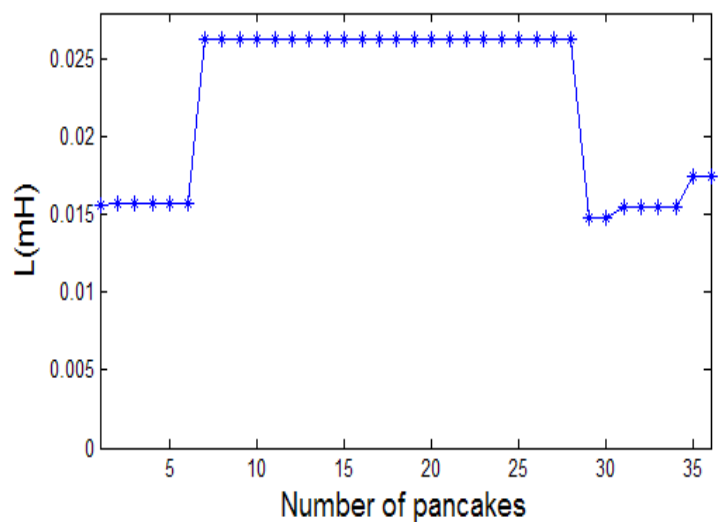

Fig. 8. Self-inductances of all units

\section{CAlculation Results}

The nodal equation of the equivalent circuit can be expressed as follows.

$$
C_{n} \frac{d^{2} u_{t}}{d t^{2}}+L_{n} u_{t}=C_{r} \frac{d^{2} e_{t}}{d t^{2}}+L_{r} e_{t}
$$

where, $u_{t}$ is the column vector of the voltage, $\boldsymbol{C}_{n}$ is the nodal capacitance matrix, $\boldsymbol{L}_{n}$ is the nodal inverted inductance matrix, $\boldsymbol{C}_{r}$ and $\boldsymbol{L}_{r}$ respectively are right column vectors of the capacitive and inductive branches, and $e_{t}$ is the impulse wave voltage source, the winding resistance is ignored.

The coefficient matrix in (1) is symmetric positive definite which satisfies the generalized eigenvalue problem. Therefore, the generalized eigenvalue method of solving coefficient matrix can be used to solve this problem.

\section{A. Voltage distribution under full lightning wave}

According to the equivalent model shown in Fig. 2, the standard full and chopped lightning-impulse voltages are respectively excited to the AC winding of FCL. Under the standard full lightning-impulse voltage, the electrical potential distribution of all the pancakes at the moment, $0.8 \mu \mathrm{s}$, is depicted in Fig. 9. It can be seen that the voltage distribution of AC windings of HTS FCL is extremely uneven under the condition of impulse voltage excitation. Figs. 10 and 11 show electric potential of the $5^{\text {th }}$ and $50^{\text {th }}$ pancakes, respectively. The transient electric potential of pancakes fluctuates due to the interaction of inductances and capacitances.

The maximum voltage of oil ducts is shown in Fig. 12. The p. u. peak electric potential gradient, about $7.08 \%$ under full lightning wave, is located at the $27^{\text {th }}$ oil duct. The voltage drop can be calculated as $950 \times 7.08 \%=67.26 \mathrm{kV}$, comparison with the permitted voltage threshold of the oil duct.

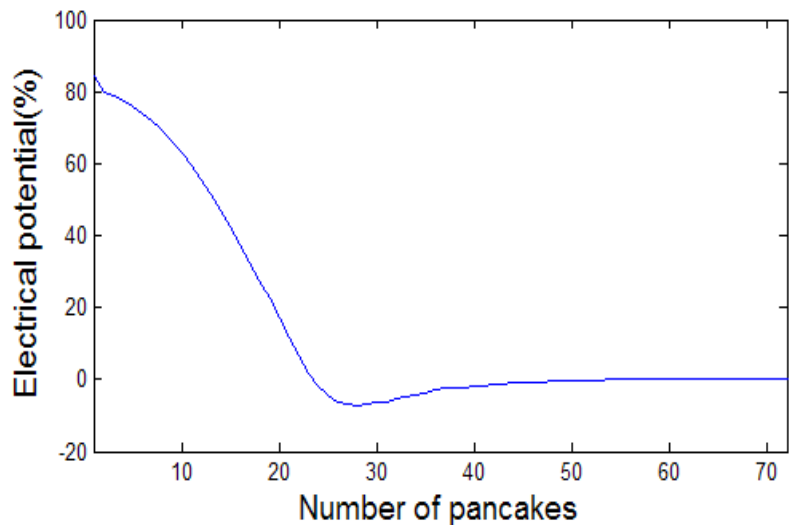

Fig. 9. Voltage distribution of all pancakes at the moment at $0.8 \mu \mathrm{s}$

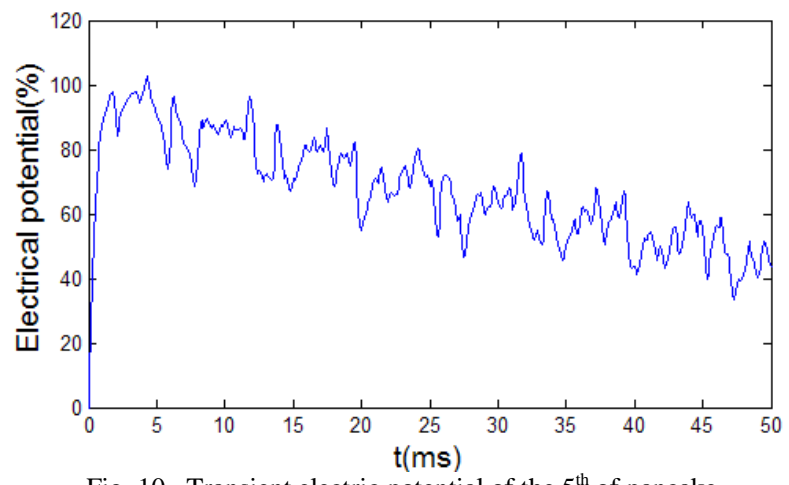

Fig. 10. Transient electric potential of the $5^{\text {th }}$ of pancake

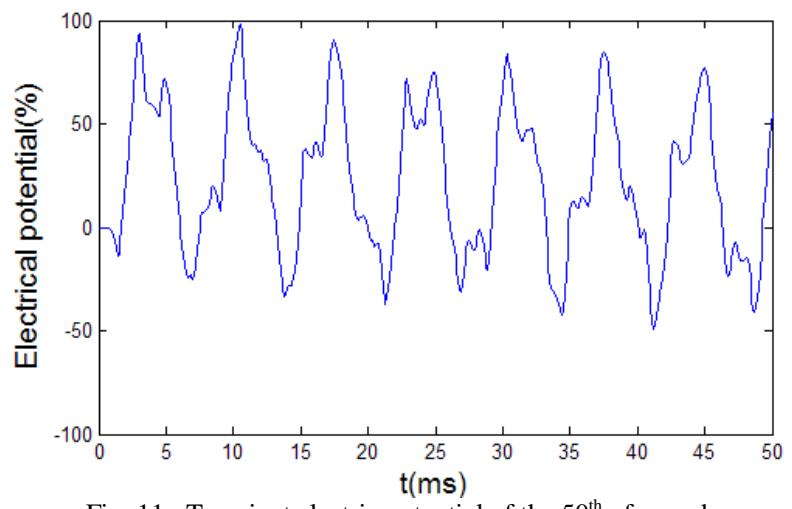

Fig. 11. Transient electric potential of the $50^{\text {th }}$ of pancake 


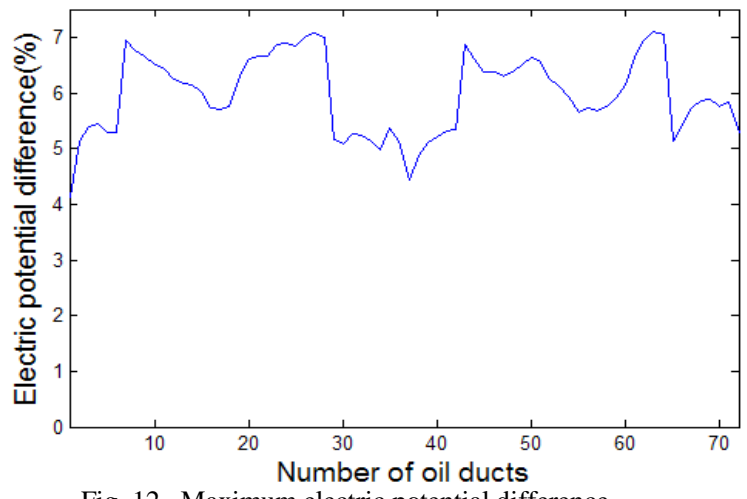

Fig. 12. Maximum electric potential difference

\section{B. Voltage distribution under chopped lightning wave}

Excited by the standard chopped lightning-impulse voltage, the electrical potential distribution of all the pancakes at the moment, $1.1 \mu \mathrm{s}$, is illustrated in Fig. 13. The voltage distribution of AC windings of HTS FCL is extremely uneven under the standard chopped lightning-impulse voltage. Figs. 14 and 15 show electric potential of the $5^{\text {th }}$ and $50^{\text {th }}$ pancakes, respectively. The maximum voltage of oil ducts is shown in Fig. 16. $27^{\text {th }}$ oil duct has the peak electric potential gradient about $7.72 \%$ under chopped lightning wave as same as full wave. The voltage drop can be calculated as $1050 \times$ $7.72 \%=81.06 \mathrm{kV}$, comparison with the permitted voltage threshold of the oil duct.

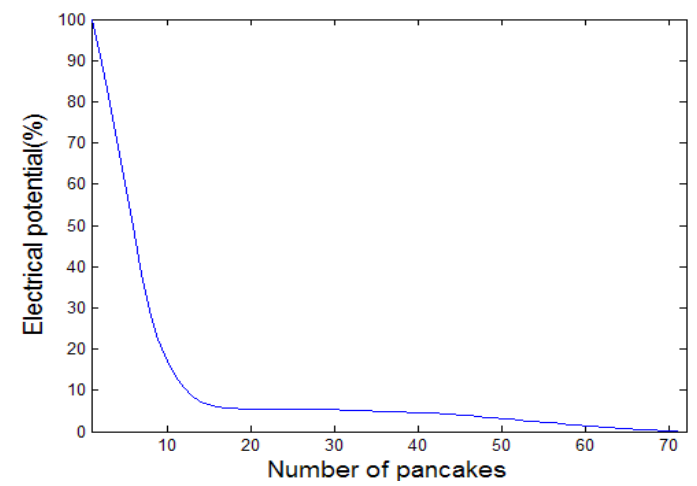

Fig .13. Voltage distribution of all pancakes at the moment at $1.1 \mu \mathrm{s}$

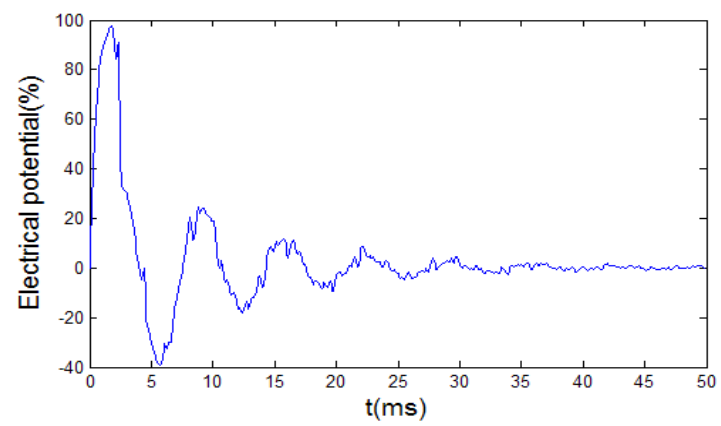

Fig. 14. Transient electric potential of the 5 th of pancake

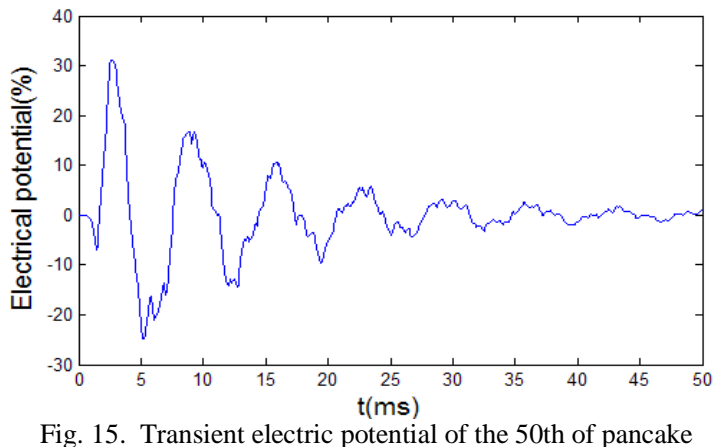

Fig. 15. Transient electric potential of the 50th of pancake

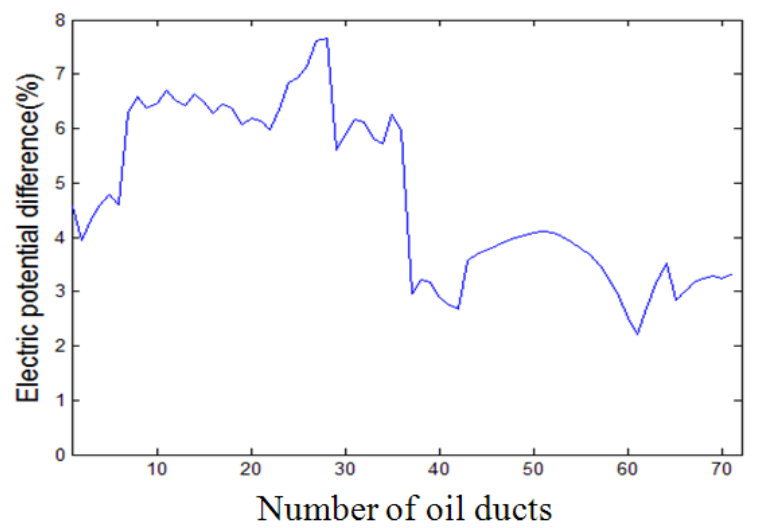

Fig. 16. Maximum electric potential difference

\section{CONCLUSION:}

This paper presents the voltage calculation of saturated core type HTS FCL under transient overvoltage. The distribution of electric potential and electric potential difference in the cases of full and chopped lightning-impulse voltage are analyzed. Both maximum electric potential gradient under full wave and chopped wave exists in $27^{\text {th }}$ oil duct. The weaknesses of minor insulation exist around $27^{\text {th }}$ oil duct in AC winding.

\section{REFERENCES}

[1] Ying Xin, Weizhi Gong, Xiaoye Niu, Zhengjian Cao, Jingyin Zhang "Development of Saturated Iron Core HTS Fault Current Limiters", IEEE Trans on Applied Superconductivity, vol. 17, June 2007, pp.17601763.

[2] T. Kataoka and H.Yamaguchi, "Comparative study of transformer type superconducting fault current limiters considering magnetic saturation of iron core," IEEE Trans. Magnetics, vol. 42, October 2006, pp. 33863388.

[3] Fan Kai, Jie Qiu, Shuhong Wang, Fang Xu, et al. "Transient Simulation and Analysis for Three-Phase Saturated Core High Temperature Superconducting Fault Current Limiter”. CEFC 2008, Athens, Greece, 2008, pp. 503.

[4] Jiaxiang Zhang, "Winding Wave Process of Transformer", China Water Power Press, 1982, pp.78-135.

[5] Woivre, V. , Arthaud, J.P., Ahmad, A., Burais, N., "Transient overvoltage study and model for shell-type power transformers”, IEEE Transactions on Power Delivery, Jan 1993, pp.212-222.

[6] Zanji Wang, "Fast Transient Modeling of Transformer Windings", Proceeding of the CSEE, 1996,16(5), pp.299-305. 